FOUNDATIONS OF COMPUTING AND DECISION SCIENCES

Vol. 38

(2013)

No. 3

DOI: $10.2478 /$ fcds-2013-00ロ 0

ISSN 0867-6356

e-ISSN 2300-3405

\title{
SCENARIO PLANNING + MCDA PROCEDURE FOR INNOVATION SELECTION PROBLEM
}

\author{
Jerzy MICHNIK *
}

\begin{abstract}
Selecting the proper set of innovation projects is the vital point of the innovation management in a firm. To make a good decision, managers have to consider many conflicting objectives in a highly uncertain environment. In this article, the comprehensive decision procedure based on scenario planning and multiple criteria analysis is presented. The main part of the procedure is a two-step model in which Hurwicz's rule and VIKOR-S method are employed to gain the selection of alternatives. To build up the decision base, the classification of the criteria involved in the innovation project selection into five categories is proposed.
\end{abstract}

Keywords: innovation management, multiple criteria decision analysis, scenario planning, uncertainty, VIKOR, VIKOR-S

\section{Introduction}

Innovation means to manufacture something new or to operate in a new way. Innovation management is a continuous and complicated process that pertains to a majority of a firm's activities and engages the significant part of firm's resources. It also goes far beyond the organizational boundaries. The importance of innovation management is widely recognized among academics and practitioners, as the innovations play the crucial role in building a sustainable competitive advantage of a firm and can contribute significantly to a firm's growth and profitability $[9$, p. 100] $[22$, p. 5] [23, p. xxxvii] [7, 19]

In innovation management, a major decision concerns the selection of some limited number of innovation projects from the initially larger set of potential projects. This decision is characterized by many conflicting objectives. Despite the critical role of innovation management and the abundance of literature discussing the role of innovation management, articles about the MCDA applications are much more modestly

*email: jerzy.michnik@ue.katowice.pl; University of Economics in Katowice ul. 1 Maja 50, 40-287 Katowice, Poland 
represented in the academic journals. The AHP method is the exception as it has become popular in practical applications $[1,10]$. Also a few other MCDA approaches have been applied in large manufacturing firms $[13,15]$.

Uncertainty is another critical issue in managing the innovation projects. In the chapter entitled "Dealing with Uncertainties in MCDA" Stewart [21] presented the review of various approaches. The majority of MCDA models that deal with uncertainty are characterized by strong assumptions and are quite complicated. In particular, the models integrating MCDA and scenario planning (SP) are considered convenient tools in solving strategic problems $[11,14]$. As the innovation management is closely related to strategic management, the integrated SP and MCDA approach can also make the foundation for decision aiding procedure in managing innovations.

In this article the comprehensive procedure for selecting the innovation projects for further processing is presented. The two-step SP+MCDA model is the main part of this procedure. To deal with uncertainty a number of scenarios are developed. In the first step of the model, the Hurwicz's rule is used for evaluating alternatives across the scenarios. In the second step, the method based on the relation of alternatives to the reference point has been employed to rank the alternatives. For this task, the variant of VIKOR method has been developed. While the original VIKOR aimed at the choice of the best alternative, it aims for sorting the alternatives into two categories: selected for development and abandoned, To underline the 'selection' goal the method has been given the working name VIKOR-S.

The remaining part of the article is organized as follows: In the next section (sec. 2) the decision process of selecting innovation projects is introduced. Sec. 3 contains the presentation of the proposed decision procedure and its application to a hypothetical innovation selection problem. The last section concludes the analysis.

\section{Decision process in innovation management}

In a series of publications devoted to the new product management Cooper et al. [3-6] strongly advocate the continuous decision process in which the selection of new product development projects plays the crucial role. This statement has been confirmed by Scott [20]. Academic and industry participants which took part in his extended survey indicated "strategic planning for technology products" and "new product project selection" as the most important among top 24 technology management issues.

\subsection{Criteria in innovation management}

The numerous criteria of various nature (qualitative and quantitative) and position (strategic, operational) are involved in the decision making for innovation management. They can represent the main (strategic) goals like successful business performance [3], product performance (profitability, sales) [8], sustainable competitive advantage [9, p. 100], or they can go down to the operational level and represent various aspects of firm's operations. 
The reports of practical applications of MCDA models in the innovation management bring several examples of criteria which can be located at the different places of strategic and/or operational level $[1,10,13,15]$. Customer satisfaction [10] and lifecycle stage of a product [13] definitely have the external nature. On the other hand, some criteria are connected with various internal activities of the firm. The examples are: fit with firm's core technological competencies [1], employee satisfaction [10].

In most cases, however, the criteria do not fit to the simple internal-external opposition. All kinds of financial criteria can serve as good examples. The investment cost in a project depends not only on the financial situation of the firm (cash resources) but also on the general economic situation (prices) and interest rates (if an investment is to be financed with external resources). Consequently, it is more appropriate to classify the criteria according to their managerial content. We propose five general categories:

- strategic criteria, designed to represent the strategic plans and long term goals,

- organizational criteria comprising organizational, marketing, logistic and other similar competencies,

- technological criteria, connected with R\&D potential and technological competencies,

- financial criteria representing investment costs and potential revenues,

- market criteria encompassing the broad range of market factors, including customers' and competitors' reaction to a new product.

\subsection{Uncertainty in innovation management}

Facing rapid globalization and commoditization, firms need to innovate constantly in order to sustain competitive advantage $[23$, p. 3]. But the innovation management is characterized by uncertain and changing information [3]. The risk built-in the innovation development results in low success rates and many projects are terminated midway in the development process [7]. The rate of successful projects which achieve the market is very low [5].

There are several sources of uncertainty in innovation management. Crucial innovations (new core products) need usually considerable changes in technology and/or organization and demand high investments. The considerable uncertainty comes from the market. It is difficult to predict precisely the customers and competitors reaction to a new product. These both uncertainties have significant impact on product performance.

\subsection{The research goal}

In innovation management, the most common decision problem is to select a subset of projects from usually much more numerous set of preselected feasible projects. 
The number of projects in the selected subset should be matched with the available resources. The ranking of projects built up according to a chosen decision rule can be a helpful tool in a decision process. We assume that as a result of the initial selection the innovation projects are independent and not mutually exclusive. In this case, the DM is not interested in eliminating the dominated alternatives as they can still be more preferred then some other non-dominated alternatives.

The main research goal is to develop adequate model to support the project selection process in innovation management. Such a model has to comprise suitable set of criteria and take into account uncertainty. The innovations projects will play the role of decision alternatives.

\subsection{Scenario planning and MCDA}

Combined SP+MCDA models seem to be an attractive alternative for the decision aid in the field of innovation management. This proposition is supported by at least three reasons:

- Scenario planning is commonly used in strategic management to which innovation management is strongly related.

- Scenario planning gives wide overview of uncertain and uncontrollable external factors which may impact on consequences of the strategic decisions.

- Scenario planning has a qualitative profile and is less demanding in strict assumptions (completeness) and parameter assessment (probabilities).

According to Stewart [21] who discusses the possible integration of scenario planning and MCDA, two approaches are apparent. The first one - called Model A - is: "apply a standard MCDA approach to construct a preference model across all $\mathrm{ms}$ possible outcomes (combinations of $m$ alternatives and $s$ scenarios)". This results in the aggregate performance measures of each alternative under each scenario. The method requires the next step: the selection of the alternative which is most preferred across all scenarios.

The second apparent approach - called Model B is: "treat each of the $n s$ criterionscenario combinations as metacriteria ( $n$ - the number of original criteria), and apply some form of MCDA to the problem of comparing $m$ alternatives in terms of the $n \times s$ metacriteria". This may generate a serious problems in a situation in which the number of criteria and number of scenarios are quite big.

In principle, nothing imposes the order of evaluation in two-step model. It is why we propose to extend this classification by splitting the model A into two. The model described above we will call model A1 and we introduce the other one, called A2, which is characterized by the reversed order of evaluations. In the first step the evaluation of alternatives across scenarios is performed. Then, in the second step, the MCDA method is chosen to evaluate alternatives in relation to criteria. 


\section{Dealing with uncertainties - multicriterial procedure for in- novation management}

We propose the decision support procedure consisting of 7 stages:

Stage 1 The suitable set of criteria is developed on the base of main objectives and important strategic issues. The DM set up the weights that represents his opinion on relative importance of criteria.

Stage 2 The limited number of scenarios (2-5) is evolved.

Stage 3 Taking into account the rigid constraints, the preliminary set of potential alternatives is selected.

Stage 4 In each scenario, the values of all criteria are assigned to alternatives. The values are taken from a predefined scale and can be assigned directly by DM or by experts.

Stage 5 Evaluation of alternatives across scenarios (1st step of the SP+MCDA model).

Stage 6 MCDA evaluation (2nd step of the SP+MCDA model).

Stage 7 The analysis of the resulting ranking and final decision.

\subsection{Basic assumptions and notations}

We consider a finite number of alternatives $A_{i}, i=1, \ldots, I$ which are evaluated by finite number of criteria $C_{j}, j=1, \ldots, J$. The evaluations may differ depending on which scenario $S_{k}$ from $K$ different scenarios will be realized. In each case, the DM is asked to assign the value $v_{i j k}$ from the common scale (e.g. 0-100), for both qualitative and quantitative criteria, where 0 is the worst and 100 is the best value (it follows that all criteria are to be maximized). The DM has also to assign the non-negative weights to all criteria (also from the same scale). Such scoring method is considered as convenient for DM and popular in MCDA models (see e.g. [11, 14]).

To illustrate our approach we consider a hypothetical problem of innovative manufacturer who is to decide about the development of new products. During last years the firm limited itself to the incremental innovations and minor improvements of their products. So, now the firm faces the need to set up the new portfolio of innovation projects. The main task is to select the subset containing the best candidates among the number of feasible projects.

In the following subsections we illustrate stage by stage the procedure presented at the beginning of this section. 


\subsection{Example of proposed procedure}

\subsubsection{Stage 1}

At the beginning a user needs to decide which and how many criteria are important for the task. For the sake of simplicity each criteria category mentioned in Sec. 2.1 will be represented by only one criterion (such a representative criterion can also be thought as the cumulative criterion comprising the valuation of a number of criteria from a given category), i.e.:

- strategic: consistency with strategic plans $\left(C_{1}\right)$,

- organizational: organizational competencies $\left(C_{2}\right)$,

- technological: technological competencies $\left(C_{3}\right)$,

- financial: financial performance $\left(C_{4}\right)$,

- market: response of the market $\left(C_{5}\right)$.

Using the common scale 0-100, the DM assigned the relative weights to all criteria. Let's assume that technological competencies and financial performance have been recognized as the most important and received weights 80 and 75 accordingly. Consistency with strategic plans received medium score while response of the market and organizational competencies have been placed at the bottom of the list. The weights of all criteria are shown in parenthesis in the top line of Table 1.

\subsubsection{Stage 2}

Generally, scenarios should cover the range of the key uncertainties which arise from the problem analysis. They should also provide a comprehensive and challenging framework against which the DM can develop and test strategies and action plans [12]. In this example we limit ourselves to three scenarios which is enough for the exemplification of our approach. They represent the anticipated general scenarios of the market development: $S_{1}$ - stability, $S_{2}$ - increase, and $S_{3}$ - decrease.

\subsubsection{Stage 3}

After analysis of rigid constraints including all kinds of available resources the 7 new innovation projects have been chosen as the alternatives $\left(A_{1}, \ldots, A_{7}\right)$. They differ in the innovation level, technological complexity, potential investment cost and return, and market response. 


\begin{tabular}{cccccccccccccccc}
\hline Criteria & \multicolumn{3}{c}{$C_{1}(65)$} & \multicolumn{3}{c}{$C_{2}(40)$} & \multicolumn{3}{c}{$C_{3}(80)$} & \multicolumn{3}{c}{$C_{4}(75)$} & \multicolumn{3}{c}{$C_{5}(55)$} \\
\hline Scenarios & $S_{1}$ & $S_{2}$ & $S_{3}$ & $S_{1}$ & $S_{2}$ & $S_{3}$ & $S_{1}$ & $S_{2}$ & $S_{3}$ & $S_{1}$ & $S_{2}$ & $S_{3}$ & $S_{1}$ & $S_{2}$ & $S_{3}$ \\
\hline$A_{1}$ & 65 & 80 & 55 & 80 & 75 & 75 & 45 & 55 & 60 & 95 & 90 & 75 & 68 & 62 & 55 \\
$A_{2}$ & 75 & 75 & 80 & 88 & 83 & 83 & 50 & 55 & 50 & 55 & 50 & 35 & 70 & 66 & 60 \\
$A_{3}$ & 70 & 85 & 65 & 50 & 40 & 40 & 55 & 60 & 60 & 85 & 80 & 75 & 73 & 70 & 65 \\
$A_{4}$ & 90 & 80 & 75 & 80 & 75 & 75 & 75 & 80 & 82 & 48 & 42 & 39 & 75 & 72 & 66 \\
$A_{5}$ & 80 & 75 & 75 & 60 & 66 & 60 & 62 & 55 & 50 & 73 & 68 & 62 & 73 & 67 & 65 \\
$A_{6}$ & 80 & 85 & 80 & 92 & 95 & 92 & 77 & 85 & 80 & 43 & 40 & 35 & 80 & 78 & 75 \\
$A_{7}$ & 66 & 58 & 55 & 80 & 72 & 65 & 67 & 73 & 70 & 50 & 45 & 35 & 68 & 75 & 60 \\
\hline
\end{tabular}

Table 1: Input values $v_{i j k}$ for alternatives $A_{i}$ and weights for criteria (in parenthesis).

\subsubsection{Stage 4}

Let's consider the example of assignment of scores for alternative $A_{1}$. Assuming that it is big innovation with high possible return especially with stable and growing market. Yet, it needs to acquire more technological competencies and the market response is not anticipated as very positive for a firm. Accordingly this project received the high scores for financial performance: 95 for stable market scenario, 90 for growing market and 75 for decreasing, but medium scores (45-60) for fit to technological competencies. In similar way the all other scores for considered projects need to by assigned. The values chose for our example are presented in Table 1.

\subsubsection{Stage 5}

For each alternative we select the criterion and consider its different outcomes in different scenarios. Following the old idea of Hurwicz (see e.g [18, p. 6-19]), we can define the following values

$$
h_{i j}=\alpha \min _{k} v_{i j k}+(1-\alpha) \max _{k} v_{i j k}, i=1, \ldots, I, j=1, \ldots, J .,
$$

where the parameter $\alpha \in[0,1]$ represents the DM's propensity towards risk (the extremes are: $\alpha=1$ for pessimistic DM, $\alpha=0$ for optimistic DM).

To continue numerical analysis of our example we assumed that the DM is rather pessimistic and we set $\alpha=0.7$. Applying the formula (1) we receive the values $h_{i j}$ (Table 2).

\subsubsection{Stage 6}

There are a plenty of various MCDA models that can be applied at the second step. The proper choice of the method should take into account not only the underlying problem structure, but also the DM view of the problem and the proposed approach.

We assume the dependence between the criteria can be neglected and we can use any method based on the weighted sum aggregation. Of course this assumption can be abandoned at any time and other suitable method which can deal with criteria 


\begin{tabular}{cccccc}
\hline & $h_{i 1}$ & $h_{i 2}$ & $h_{i 3}$ & $h_{i 4}$ & $h_{i 5}$ \\
\hline$A_{1}$ & 62,5 & 76,5 & 49,5 & 81,0 & 58,9 \\
$A_{2}$ & 76,5 & 83,0 & 50,0 & 41,0 & 63,0 \\
$A_{3}$ & 71,0 & 40,0 & 55,0 & 78,0 & 67,4 \\
$A_{4}$ & 79,5 & 75,0 & 75,0 & 41,7 & 68,7 \\
$A_{5}$ & 76,5 & 60,0 & 50,0 & 65,3 & 67,4 \\
$A_{6}$ & 81,5 & 92,0 & 60,0 & 37,4 & 76,5 \\
$A_{7}$ & 58,3 & 65,0 & 60,0 & 39,5 & 64,5 \\
\hline
\end{tabular}

Table 2: The values of Hurwicz's indices $h_{i j}$ of criteria $C_{1}-C_{5}$ for alternatives $A_{i}$.

dependence can be applied. The approaches that lead to outranking relations are also possible if they appear to better reflect the DM preferences.

For our task we have chosen the modified VIKOR method taking into account some of its advantages $[16,17]$ :

- ranking alternatives according to their closeness to the ideal solution,

- seeking a compromise between two views: average of all criteria point of view (linear metrics) versus the weakest criterion point of view (Tchebyshev metrics).

VIKOR has been successfully applied in the problems regarding innovation. With the aid of VIKOR Wu et. al. determined a ranking of various types of Taiwanese universities based on indicators of innovation capital [24]. It has been also adopted for the construction of a novel aviatic innovation system [2].

The VIKOR-S differs from its original by a few features. To avoid the influence of the content of feasible set on the final ranking we do not include the normalization in calculating measures $S$ and $R$ (in original procedure such normalization appears in formulas (2) and (3)). It means that the ideal solution is given by all values equal to 100 (or 1 after the conversion into decimal fractions). Consequently the anti-ideal is defined as a set of zeros.

The original VIKOR method is designated for the choice of the best compromise solution from the initial set of alternatives. In the innovation project selection it is rather common that certain number (usually more then one) of the top projects are accepted for further development. For that reason we propose to modify the $\mathrm{C} 1$ and $\mathrm{C} 2$ conditions from VIKOR procedure. This modification results in a set of compromise solutions instead of a single compromise solution.

The algorithm VIKOR-S is performed in the following steps (for convenience values and weights are converted into decimal fractions):

(a) For all alternatives compute the values of two distances from the ideal: $S_{i}$ and $R_{i}, i=1, \ldots, I$ from the following formulas

$$
S_{i}=\sum_{j=1}^{J} w_{j}\left(1-v_{i j}\right)
$$




$$
R_{i}=\max _{j}\left\{w_{j}\left(1-v_{i j}\right)\right\}
$$

where $w_{j}$ are the weights expressing the relative importance of criteria and $v_{i j}$ represents the scores that has been assigned to alternative $A_{i}$ for criterion $C_{j}$. The smaller are $S_{i}$ and $R_{i}$, the better is the alternative $A_{i}$.

(b) For all alternatives compute the values $Q_{i}, i=1, \ldots, I$ :

$$
Q_{i}=q \frac{S_{i}-S^{-}}{S^{+}-S^{-}}+(1-q) \frac{R_{i}-R^{-}}{R^{+}-R^{-}}
$$

where

$$
\begin{aligned}
S^{-}=\min _{i} S_{i}, & S^{+}=\max _{i} S_{i}, \\
R^{-}=\min _{i} R_{i}, & R^{+}=\max _{i} R_{i},
\end{aligned}
$$

and $q \in[0,1]$ is the weight that represents the importance of the "mix of all criteria" attitude against the importance of the "weakest criterion" attitude which importance is measured by $1-q$.

(c) Display the three ranking lists of alternatives, sorted by the values of $S, R$ and $Q$, in increasing order. $M_{1}$ has been chosen as a initial number of alternatives that can be carried on simultaneously.

(d) The following two conditions are satisfied:

C1 "Acceptable advantage" condition. Let

$$
Q\left(A^{\prime \prime}\right)-Q\left(A^{\prime}\right) \geq \Delta Q
$$

where $A^{\prime}$ is the last alternative from the top of the list belonging to the set of $M_{1}$ alternatives, $A^{\prime \prime}$ is the alternative with the next position in the $Q$ ranking list, after the $A^{\prime}$ alternative, $\Delta Q=1 /(I-1)$.

C2 "Acceptable stability in decision making" condition: $M_{1}$ alternatives from the top of $Q$ list occupy the first $M_{1}$ positions on at least one of two other ranking: $S$ and $R$ (this means that the set of $M_{1}$ alternatives is a stable solution at least for one of the above mentioned extreme points of view).

As a set of compromise solutions propose the $M_{1}$ alternatives from the top of the $Q$ list.

(e) Only condition C1 is not satisfied: Add to the set of $M_{1}$ alternatives the $M_{2}$ alternatives, where $M_{2}$ is a number of alternatives that fulfil condition $Q(A)-$ $Q\left(A^{\prime}\right)<\Delta Q$. The set containing $M_{1}+M_{2}$ alternatives is presented for further analysis.

(f) Only condition C2 is not satisfied: Add to the set of $M_{1}$ alternatives the $M_{3}$ items that appeared at the top $M_{1}$ positions on the $S$ and $R$ lists and that did not belong to initial set. The set containing $M_{1}+M_{3}$ alternatives is presented for further analysis. 


\begin{tabular}{ccccc}
\hline & $S$ & $Q(q=0.5)$ & $Q(q=0.6)$ & $R$ \\
\hline$A_{1}$ & 1,110 & 0,301 & 0,281 & 0,404 \\
$A_{2}$ & 1,267 & 0,709 & 0,700 & 0,443 \\
$A_{3}$ & 1,133 & 0,134 & 0,160 & 0,360 \\
$A_{4}$ & 1,043 & 0,353 & 0,282 & 0,437 \\
$A_{5}$ & 1,152 & 0,345 & 0,341 & 0,400 \\
$A_{6}$ & 1,071 & 0,542 & 0,450 & 0,470 \\
$A_{7}$ & 1,380 & 0,928 & 0,942 & 0,454 \\
\hline
\end{tabular}

Table 3: The $S, R$ and $Q$ rankings.

(g) Both conditions $\mathrm{C} 1$ and $\mathrm{C} 2$ are not satisfied: Take for further analysis all alternatives that belong to any of enlarged sets: set containing $M_{1}+M_{2}$ or set containing $M_{1}+M_{3}$ alternatives.

If the set of alternatives chosen according to the above procedure contains more then $M_{1}$ alternatives further analysis is necessary.

Now we come back to our example and use method VIKOR-S for selecting the innovation projects. We assume that initially the DM considers that the firm is able to carry 3 new projects at the same time. It means that 3 of total 7 projects have to be selected.

In our example the Hurwicz's indices $h_{i j}$ calculated in the previous stage play the role of scores $v_{i j}$. It may happen that initially the DM is indifferent to the two extreme options: an average of all criteria $(q=1)$ and weakest criterion $(q=0)$. So, we can begin the calculations with indifferent value $q=0.5$. With the aid of eq. (2) and (3) we obtain the value of $S$ and $R$. Then, from eq. (4) we calculate $Q$. The ranking calculated for $q=0.5$ is presented in Table 3. Selecting 3 projects means that the value of $M_{1}$ is 3 . The 3 top projects of the $Q$ ranking are: $A_{3}, A_{1}$ and $A_{5}$. The condition $\mathrm{C} 2$ is satisfied as top 3 alternatives in $R$ ranking are the same. However condition $\mathrm{C} 1$ is not satisfied. Alternative $A_{4}$ lies close to $A_{5}$ (the difference $Q\left(A_{4}\right)-Q\left(A_{5}\right)$ is equal 0,0076 which is less then $\left.\Delta Q \approx 0.1667\right)$. It means that the DM should consider alternative $A_{4}$ as also good enough for further analysis. If DM feels that he is more prone to the average value of all criteria then the weakest one, he can choose higher value of $q$, let say 0.6. The rankings of all alternatives for $q=0.6$ is also given in Table 3 . It can be easily seen that in this case condition $\mathrm{C} 2$ is also not satisfied. Top 3 alternatives in $Q$ ranking are: $A_{3}, A_{1}$ and $A_{4}$. But both sets of 3 top alternatives in $S$ and $R$ rankings are different from it. In $S$ ranking $A_{6}$ and in $R$ ranking $A_{5}$ are placed at the second position. According to rule (g) both $A_{6}$ and $A_{5}$ should be added to final analysis.

\subsubsection{Stage 7}

When the solution suggests more projects then it was chosen initially, the additional analysis for final selection is required to limit the final number of projects to 3 . It may also happen that DM feels that the higher number of projects (4 or 5 ) deserves to be continued. In this situation DM can consider the expanding of firm's potentiality by 
acquiring more required resources.

When the DM chooses the different value of parameter $q$, the results may change. The dependence of the aggregated outcomes of all alternatives along with the whole $q$ interval is illustrated in Fig. 1. Such a graph can be very helpful in an analysis of stability of solutions against the change of parameter $q$, especially when the DM undecided in choosing te value of $q$.

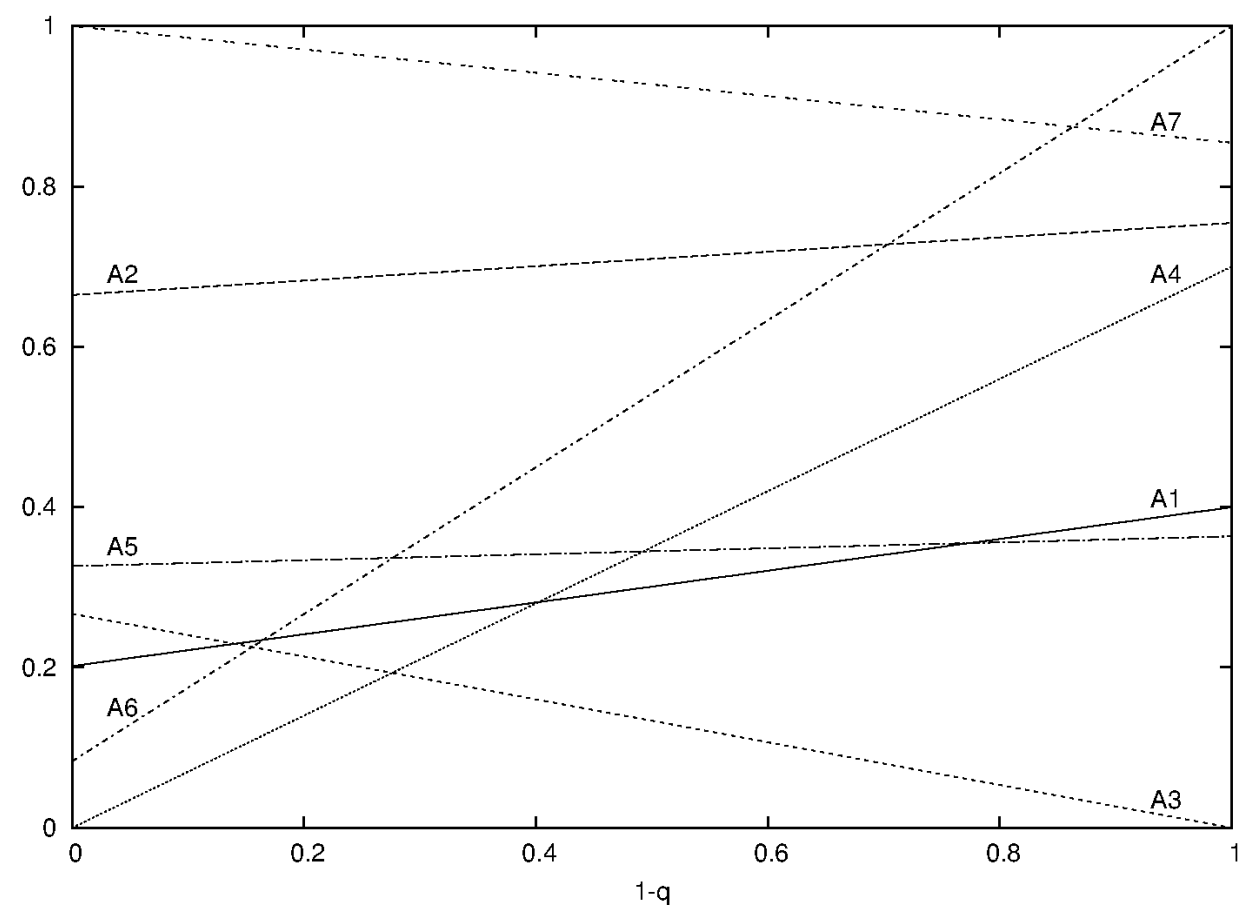

Figure 1: The dependence of the aggregated outcomes of all alternatives along with the whole $q$ interval.

\section{Conclusions}

Selecting the limited number of innovation projects from the preliminary larger set of potential alternatives is an important part of the innovation management and is broadly discussed in the monographs and articles. Although the informal approaches dominate in literature, there are few examples of more strict decision procedures based on multiple criteria analysis. This article brings seminal procedure which can help decision making in selection of innovation projects.

The existing literature was a starting point for the analysis of criteria pertaining to the selection decision. Basing on a managerial connotation of the criteria the author 
proposed to classify them into five categories: strategic, organizational, technological, financial and market.

The decisions in the field of innovation management are considered as highly risky. This situation therefore requires the tools that can facilitate the decision making in an uncertain environment. The proposed procedure which incorporates scenario planning and multiple criteria methodology has a potential to be the useful tool for the decision aiding.

The main part of the procedure is the two-stage SP+MCDA model. In the first step, the evaluation of alternatives across scenarios with the use of Hurwicz rule is performed. For the second step the VIKOR-S method has been elaborated. VIKOR-S is the modification of VIKOR procedure and it selects the set of compromise solutions in contrast to the original method which leads to the choice of the single compromise solution.

The proposition presented in this work can be a good point of departure for the further development of efficient methods for the selection problem in innovation management and other related fields. The potential directions of future study can be:

- experiments with modifications of the model towards a better reflection of DM preferences; e.g. it may appear that in Hurwicz's formula different parameters $\alpha$ should be applied to different criteria,

- seeking for other methods that can replace those used here in the two-stage MCDA+SP model, e.g. a method which can work with dependent criteria may appear to be more suitable,

- adapting the procedure for application in other related fields; especially project management and strategic management are the most prospective fields of application.

Acknowledgements: Research partly supported by Polish Ministry of Science and Higher Education, Research Grant no. NN111 438637.

\section{References}

[1] R. J. Calantone, C. A. Benedetto, and J. B. Schmidt. Using the analytic hierarchy process in new product screening. An International Publication of The Product Development $\&$ Management Association, 16(1):65-76, 1999.

[2] Jui-Kuei Chen and I-Shuo Chen. Aviatic innovation system construction using a hybrid fuzzy MCDM model. Expert Systems with Applications, 37(12):83878394, 2010.

[3] R. G. Cooper, S. J. Edgett, and E. J. Kleinschmidt. New product portfolio management: practices and performance. Journal of Product Innovation Management, 16(4):333-351, 1999. 
[4] R. G. Cooper, Scott J. Edgett, and Elko J. Kleinschmidt. New problems, new solutions: Making portfolio management more effective. Research-Technology Management, 43:18-33, 2000.

[5] Robert G Cooper, Scott J Edgett, and Elko J Kleinschmidt. Benchmarking best NPD practices - I. Research Technology Management, 47(1):31-43, 2004.

[6] Robert G. Cooper, Scott J. Edgett, and Elko J. Kleinschmidt. Benchmarking best NPD practices - II. Research-Technology Management, 47:50-59, 2004.

[7] K. Cormican and D. O'Sullivan. Auditing best practice for effective product innovation management. Technovation, 24(10):819-829, 2004.

[8] E. Danneels and E. J. Kleinschmidt. Product innovativeness from the firm's perspective: its dimensions and their relation with project selection and performance. Journal of Product Innovation Management, 18(6):357-373, 2001.

[9] Mark Dodgson, David Gann, and Ammon Salter. The Management of Technological Innovation. Oxford University Press, 2008.

[10] R. L. Englund and R. J. Graham. From experience: linking projects to strategy. Journal of Product Innovation Management, 16(1):52-64, 1999.

[11] Paul Goodwin and George Wright. Enhancing strategy evaluation in scenario planning: a role for decision analysis. Journal of Management Studies, 38(1): $1-16,2001$.

[12] Kees van der Heijden. Scenarios: The Art of Strategic Conversation. Wiley, 1 edition, 1996.

[13] J. D. Linton, S. T. Walsh, B. A. Kirchhoff, J. Morabito, and M. Merges. Selection of R\&D projects in a portfolio. In Engineering Management Society, 2000. Proceedings of the 2000 IEEE, pages 506-511, 2000.

[14] Gilberto Montibeller, Haidee Gummer, and Daniele Tumidei. Combining scenario planning and multi-criteria decision analysis in practice. Journal of MultiCriteria Decision Analysis, 14(1-3):5-20, 2006.

[15] M. S. Morcos. Modelling resource allocation of R\&D project portfolios using a multi-criteria decision-making methodology. International Journal of Quality and Reliability Management, 25(1):72-86, 2008.

[16] S. Opricovic, S., . Multicriteria Optimization of Civil Engineering Systems, Technical Report, Belgrade: Faculty of Civil Engineering, 1998.

[17] Serafim Opricovic and Gwo-Hshiung Tzeng. Compromise solution by MCDM methods: A comparative analysis of VIKOR and TOPSIS. European Journal of Operational Research, 156(2):445-455, 2004.

[18] A. Ravi Ravindran, editor. Operations Research and Management Science Handbook. 2008. 
[19] Sören Salomo, Joachim Weise, and Hans Georg Gemünden. NPD planning activities and innovation performance: The mediating role of process management and the moderating effect of product innovativeness. Journal of Product Innovation Management, 24(4):285-302, 2007.

[20] George M. Scott. Critical technology management issues of new product development in high-tech companies. Journal of Product Innovation Management, 17 (1):57-77, 2000 .

[21] T.J. Stewart. Dealing with uncertainties in MCDA. In J. Figueira, S. Greco, and M. Erghott, editors, Multiple Criteria Decision Analysis, State of the Art Surveys., pages 445-470. Springer Science + Business Media, 2005.

[22] Paul Trott. Innovation management and new product development. Pearson Education, 2005.

[23] J. Christopher Westland. Global Innovation Management. Palgrave, Macmillan, 2008.

[24] Hung-Yi Wu, Jui-Kuei Chen, and I-Shuo Chen. Innovation capital indicator assessment of taiwanese universities: A hybrid fuzzy model application. Expert Systems with Applications, 37(2):1635-1642, 2010.

Received October, 2012 